\title{
Modified Wavelet Transform Features for Characterizing Different Plaque Types in IVUS Images; A Feasibility Study
}

\author{
A. Roodaki ${ }^{1}$, A. Taki ${ }^{1,2}$, S. K. Setarehdan ${ }^{1}$, N. Navab ${ }^{2}$ \\ ${ }^{1}$ Control and Intelligent Processing Center of Excellence, Faculty of Electrical and Computer \\ Engineering, University of Tehran, Tehran, Iran. \\ ${ }^{2}$ Computer Aided Medical Procedures (CAMP) - TU Munich, Germany \\ ksetareh@ut.ac.ir
}

\begin{abstract}
Atherosclerosis is a leading cause of most cardiovascular diseases. Current intravascular ultrasound (IVUS) image processing techniques do not allow adequate and effective identification of the coronary artery plaques. This can be improved by defining more discriminative features for each kind of artery plaques. In this paper, the effectiveness of a modified wavelet transform feature extraction method and the Gabor filter were studied for automated characterization of the atherosclerosis plaques within the IVUS images. The methods are applied on 100 IVUS images obtained from five different patients. Support vector machine was employed in the classification step. As a result an accuracy rate of about $80 \%$ was achieved for all methods.
\end{abstract}

\section{Introduction}

Cardiovascular diseases caused by atherosclerosis are the leading cause of mortality in the developed countries. Lesion components respond differently to pharmaceuticals and interventional therapies; therefore differentiating between various tissue and plaque types is of paramount importance. Common clinically important plaque types include: Calcium, Fibrous, Necrotic, and Lipid types [1]. The size of each plaque type in the plaque area and also their combination define the type of the problem $[1,2]$.

The most frequent cause of coronary thrombosis is a thin fibrous cap heavily covering an underlying lipidic core that is in communication with the flowing blood $[1,2]$. In addition, focal calcifications often form the initiating site of dissection after balloon angioplasty and specific patterns of calcification strongly correlate with the risk of major postangioplasty dissection [3]. Therefore, accurate characterization of the plaque lesions helps to choose the appropriate clinical intervention and assess the therapy effects.

High-resolution intravascular ultrasound (IVUS) has provided clinically important means to demonstrate the extent and distribution of the coronary artery disease and reveal the severity and eccentric nature of the plaque lesions which may be underestimated in angiography. IVUS has also provided insights into the plaque distribution and the extent of the lipid-rich plaques, calcification and thickness of the fibrous cap.

The tedious and time-consuming task of manual processing and interpretation of IVUS images suffers from intra- and interobserver variability. Furthermore, an expert may not be able to completely characterize the different compositions of the atherosclerotic plaques. Thus, automatically characterizing the IVUS images is demanding and challenging.

Several methods have been proposed for characterizing atherosclerosis composition using IVUS technique recently [4-7]. Generally, there are two approaches for analyzing the IVUS data: the first approach is to examine the IVUS image by extracting the important textural features and to assign each pixel into one of the predefined classes [4,5].

The second approach is to analyze the ultrasound RF signals directly rather than using the IVUS images generated from them [6,7]. Since only the amplitude information of the RF signals is used for generating the IVUS images, the second method is superior. In [6,7], a novel method based on the power spectral analysis of the RF signals has been proposed which is known as the "Virtual Histology (VH)". The in vivo study of the IVUS-VH method is reported to be highly correlated with those of histopathology [8,9]. Different 
investigations showed the efficacy of this method for classification of different types of coronary plaque components so $\mathrm{VH}$ is a widely accepted and extensively used clinical method [8,9]. Perhaps the only limitation of this technique is the large storage needed for saving the IVUS backscattered signal of every subject.

In this paper, the efficiency of the wavelet transform (with two different mother wavelet functions) and also the Gabor filter for characterizing different plaque types in IVUS images is investigated. Wavelet transform provides both time and frequency domain analysis of the signals and images. The Gabor filter is a kind of wavelet transform. These methods have been extensively used in image processing and pattern recognition researches in the past $[5,10,11]$. These methods provide a precise and unifying framework for the analysis and characterization of a signal at different scales.

The Support Vector Machine (SVM) is employed for classifying the extracted feature vectors. SVM is known to have more reliable results in comparison to other classifiers such as neural networks. It has outperformed the traditional techniques in various applications [10]. There are four classes of calcium, fibrous, fibrolipidic, and necrotic core in IVUS-VH. In $[6,7]$ all areas that exhibited mild to heavy lipid accumulation were diagnosed as fibrolipidic. Thus, the fibrolipidic areas exhibit a large variation in intensity levels making it hard to classify all four plaque types at just one step. Therefore, we unify the fibrous and fibrolipidic classes in this work producing three classes in total. To ensure the comparability of the results and validity of the comparisons, the same borders that were detected in the VH images are used in this study.

The rest of the paper is organized as follows. Section 2 describes the proposed methods for characterizing plaque compositions in IVUS images. Section 3 compares the results obtained using the proposed method to those of other methods. Finally section 4 concludes the paper.

\section{Methodology}

\subsection{Wavelet transform:}

Wavelet transform provides a time-frequency representation of a signal. Wavelet coefficients of a signal, $f(t)$, are the projections of the signal onto the multiresolution subspace $V_{j}=\operatorname{span}\left\{\varphi_{j, k}(t), k \in Z\right\}$ and $W_{j}=\operatorname{span}\left\{\psi_{j, k}(t), k \in Z\right\}, j \in Z$ where the basis functions $\varphi_{j, k}(t)$ and $\psi_{j, k}(t)$ are constructed by dyadic dilations and translations of the scaling and wavelet functions $\varphi(t)$ and $\psi(t)$ as follows:

$\varphi_{j, k}(t)=2^{j / 2} \varphi\left(2^{j} t-k\right)$

$\psi_{j, k}(t)=2^{j / 2} \psi\left(2^{j} t-k\right)$

where $j$ is the scaling parameter and $k$ is the translation parameter. The wavelet decomposition of a 2-D signal (image) can be achieved by applying the 1-D wavelet decomposition along the rows and columns of the image separately. This is equivalent to projecting the image onto separable 2-D basis functions obtained from the products of 1-D basis functions. First, in each level, images are decomposed into four sub-images: LL, LH, HL, and HH. The sub-band LL (low frequency content) is used for the decomposition into the further decomposition levels.

In this research, we have averaged the sub-images in the horizontal and vertical directions for achieving more robustness in the aspect of being invariance to rotation. The reason for this is the fact that there are many structural variations in each plaque type in IVUS images. Finally, the energy function for each sub-image is calculated and used as a feature. Therefore, the number of the features would count to $2 * \mathrm{~N}+1$, where $\mathrm{N}$ is the maximum number of decomposition levels.

\subsection{Gabor filter}

A Gabor Filter is a special case of wavelets. It is essentially a Gaussian function which is modulated by a complex sinusoid. A 2-D Gabor filter in the spatial domain can be considered as follows:

$G(x, y ; S d, f, \varphi)=\exp \left(-\frac{x^{\prime 2}+y^{\prime 2}}{2 S d}\right) \times \cos \left(2 \Pi f x^{\prime}\right)$

where

$x^{\prime}=x \cdot \cos (\varphi)+y \cdot \sin (\varphi)$

$y^{\prime}=y \cdot \cos (\varphi)-x \cdot \sin (\varphi)$.

The performance of the Gabor filter depends on the selection of the input parameters correctly.

\subsection{Support Vector machines}

In this section, we will briefly review the use of SVMs in classification problems. For further knowledge on SVMs one can refer to [10]. Let the training set $D$ to be represented by $\left\{\left(x_{i}, y_{i}\right)\right\}^{N}{ }_{i=1}$, with each input $x_{i} \in \mathfrak{R}^{m}$ and the output label assuming two classes $y_{i} \in\{ \pm 1\}$. The SVM first maps $x$ to $z=\varphi(x)$ in a Hilbert space $F$ (with inner product $<\varphi($.), $\varphi($.) $>$ ) via a nonlinear map $\varphi: \mathfrak{R}^{m} \rightarrow F$. The space $F$ which is usually of high dimensionality is often called the 
feature space. Considering the case when the data is linearly separable in $F$, i.e., there exists a vector $w \in F$ and a scalar $b \in \mathfrak{R}$ such that

$y_{i}\left(<w, \varphi\left(x_{i}\right)>+b\right) \geq 1$

for all elements in the training set. The SVM constructs a hyperplane $(<w, \varphi(x)>+b)$ for which the separation between the positive and negative examples is maximized.

$w$ for this optimal hyperplane can be found by minimizing $\|w\|$, and the resulting solution can be written as $w=\sum_{i=1}^{N} \alpha_{i} y_{i} \varphi\left(x_{i}\right)$ for some $\alpha_{i} \geq 0$.

The vectors $\alpha_{i}$ 's, can be found by solving the quadratic programming problem:

$\left[L_{D}=\sum_{i} \alpha_{i}-1 / 2 \sum_{i, j} \alpha_{i} \cdot \alpha_{j} \cdot y_{i} \cdot y_{j} \cdot<\varphi\left(x_{i}\right), \varphi\left(x_{j}\right)>\right]$

subject to the constraints

$\sum_{i} \alpha_{i} \cdot y_{i}=0$

$0<\alpha_{i}<C$

$i=1,2, \ldots, N$

For the non-zero elements of $\alpha_{i}$ 's, the corresponding training examples must lie along the margins of the decision boundary given by the KuhnTucker theorem. These are called support vectors. The parameter $C$ controls the tradeoff between errors of the SVM on training data and margin maximization.

Instead of mapping $\varphi\left(x_{i}\right)$ and $\varphi\left(x_{j}\right)$ explicitly first, which results in a very high dimension, SVM makes use of a kernel function $K($.$) to be substituted in (7),$ such that

$K\left(x_{i}, x_{j}\right)=<\varphi\left(x_{i}\right), \varphi\left(x_{j}\right)>$.

This approach is same for any kernel methods in general. For this property to be used, the kernel has to satisfy conditions of Mercer's Theorem. In this paper, we have used the Gaussian kernel shown in (11).

$K\left(x_{i}, x_{j}\right)=\exp \left(-\left\|x_{i}-x_{j}\right\|^{2} / 2 \sigma^{2}\right)$

The above mentioned equations were for cases where the training set is separable in the $F$ dimension. On the other hand, when the data is not separable in the $F$ dimension, like what we face here, the regularization parameter $C$, controls the tradeoff between model complexity and training error in order to ensure good generalization performance [10].

\section{Result and discussion}

The study group in this work is consisted of sequences of IVUS images acquired from five different patients. These images with the digitized matrix size of $400 \times 400$ pixels were acquired using a $30-\mathrm{MHz}$ transducer at a $0.5 \mathrm{~mm} / \mathrm{s}$ pullback speed. Out of the frames in which all types of plaques were detected by the reference VH method, 20 frames were selected for each patient. The three aforementioned methods were then applied on the set of 100 frames. The characterized IVUS images were validated by their corresponding VH images and the accuracy, sensitivity, and specificity parameters were calculated for each technique. The results of the methods were compared to each other. These values were calculated using the equations described in continue.

The area between Intima and Media-adventitia borders is scanned by a $7 \times 7$ sweeping window. Next, the aforementioned features are extracted from each sub-image in the window and assigned to the center pixel of the window. The wavelet functions used in this study were "Db1" and "Coif1". These functions are chosen as they are small enough to fit in the sub-image. The maximum number of decomposition was set to three. The Gabor filter parameters are chosen as follows:

$\mathrm{Sd}=[0.5,1,1.5,2], \mathrm{f}=[1,2,4,8], \varphi=[0,45,90,135]$.

Therefore, the number of features for wavelet analysis method was seven in comparison with the 64 features for Gabor filter method.

All the features were first normalized into $[0 \rightarrow 1]$ before classification step. The standard deviation value in SVM's Gaussian function was empirically chosen to be 0.7 . The leave-one-patient-out was used for method evaluation in this study. Table I shows the results for three plaque types. Fig. 1 illustrates an example characterized images using the Gabor filter and the "Coif1" wavelet function.

Table I. results of three used methods in this study.

\begin{tabular}{|c|c|c|c|c|c|c|c|}
\hline \multirow{2}{*}{} & \multicolumn{2}{|c|}{ Calcium } & \multicolumn{2}{c|}{ Fibrolipid } & \multicolumn{2}{c|}{ Necrotic } & \multirow{2}{*}{ accuracy } \\
\cline { 2 - 6 } & Sens. & Spec. & Sens. & Spec. & Sens. & Spec. & \\
\hline Db1 & $68 \%$ & $93 \%$ & $90 \%$ & $88 \%$ & $57 \%$ & $87 \%$ & $79 \%$ \\
\hline Coif1 & $70 \%$ & $93 \%$ & $91 \%$ & $88 \%$ & $54 \%$ & $84 \%$ & $80 \%$ \\
\hline Gabor & $64 \%$ & $83 \%$ & $92 \%$ & $84 \%$ & $48 \%$ & $89 \%$ & $78 \%$ \\
\hline
\end{tabular}



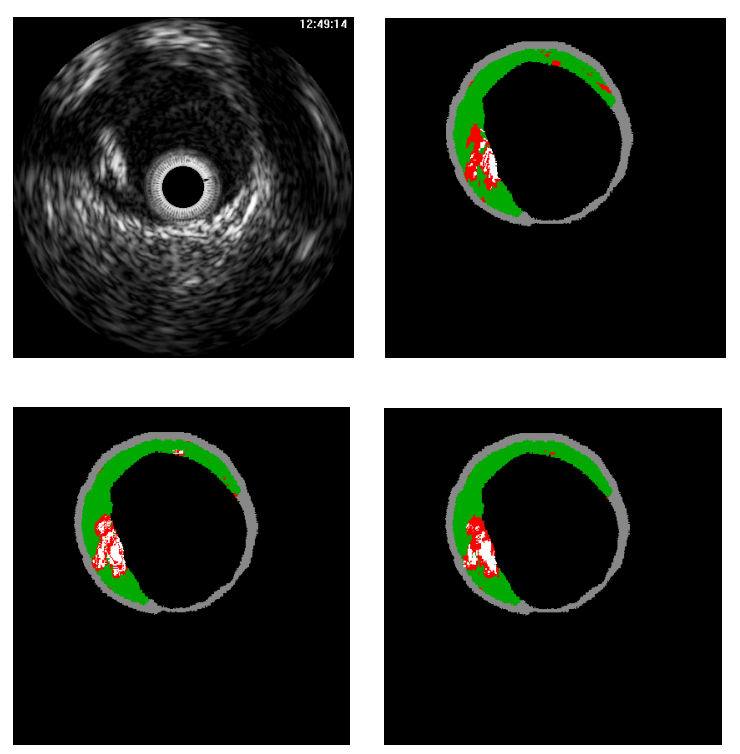

Fig. 1. From left to right: above) Original IVUS image, VH characterized image. below) Result of Gabor, Result of "Coif1". $($ Red = Necrotic, White= calcified, and Green $=$ Fibrolipid)

\section{Conclusion}

In this paper, the ability of the Gabor filter and modified wavelet transform features for characterizing arthrosclerosis plaque compositions was investigated. The SVM with a Gaussian kernel was used to classify the extracted feature vectors. The results of this study show that all three methods have somehow near accuracies. However, in overall the "coifl" wavelet transform has better performance in comparison with the other methods.

Although in signal processing methods, the frequency information of RF signal can be used along with its amplitude whereas in image processing techniques, decisions are made from the variations in the distribution of gray levels produced solely from the amplitude information of the RF signal however, the RF signals are not always available e.g. for the large dataset of images available within the archives. So, development of the image based $\mathrm{VH}$ algorithm will have a considerable impact. In fact this would allow the physicians to get the $\mathrm{VH}$ information by simply processing the previously acquired IVUS images of the patient.

\section{References}

[1] R. Virmani, F. D. Kolodgie, A. P. Burke, A. Farb, S. M. Schwartz, " Lessons From Sudden Coronary Death A Comprehensive Morphological Classification Scheme for Atherosclerotic Lesions," Arterioscler Thromb Vasc Biol., vol. 20, pp. 1262-1275, 2000.

[2] R. Viramini, A. P. Burke, F. D. Kolodgie, A. Farb, "Pathology of the Thin-Cap Fibroatheroma: A Type of Vulnerable Plaque," Journal of Interventional Cardiology, vol. 16, NO.3, 2003.

[3] Potkin BN, Keren G, Mintz GS, et al. Arterial responses to balloon coronary angioplasty: an intravascular ultrasound study. J Am Coll Cardiol, vol.20, pp: 942-951, 1992.

[4] D.G. Vince, K.J. Dixon, R.M. Cothren, J.F. Cornhill, "Comparison of texture analysis methods for the characterization of coronary plaques in intravascular ultrasound images," Computerized Medical Imaging and Graphics, vol. 24, pp. 221229, 2000.

[5] K. L. Caballero, J. Barajas, et al., "In-Vivo IVUS Tissue Classification: A Comparison Between RF Signal Analysis and Reconstructed Images", 11th Iberoamerican Congress on Pattern Recognition (CIARP06), LNCS 4225: 137-146, Cancun (Mexico) 11-2006.

[6] A. Nair, B. D. Kuban, et al. "Coronary plaque classification using intravascular ultrasound radiofrequency data analysis.," Circulation, vol. 106, pp. 2200-2206, 2002 b.

[7] A. Nair, M. P. Margolis, B. D. Kuban, and D. G. Vince, "Automated Coronoary Plaque Characterisation with IVUS Backscatter_Ex Vivo Validation", EuroIntervention 2007.

[8] G. A. Rodriguez-Granillo, E. P. Mc Fadden, M. Valgimigli, et al. "Coronary plaque composition of nonculprit lesions, assessed by in vivo intracoronary ultrasound radio frequency data analysis, is related to clinical presentation", American Heart Journal, Volume 151, Number 5, 2006.

[9] K. Nasu, E. Tsuchikane, O. Katoh, D. G. Vince, R. Virmani, et al. "Accuracy of In Vivo Coronary Plaque Morphology Assessment: A Validation Study of In Vivo Virtual Histology Compared With In Vitro Histopathology", Journal of the American College of Cardiology Vol. 47, No. 12, 2006.

[10] Shutao Lia, James T. Kwoka, et al., Texture classification using the support vector machines. Pattern Recognition 36 (2003) 2883 - 2893

[11] K. Jafari-Khouzani and H. Soltanian-Zadeh, "Rotation-Invariant Multiresolution Texture Analysis Using Radon and Wavelet Transforms", IEEE TRANSACTIONS ON Image Processing, VOL. 14, NO. 6, 2005. 